\title{
The distribution of different virulence grass carp reovirus strains in some neglected tissues
}

\author{
H.R. Liang ${ }^{1,2}$, X.Z. Fu ${ }^{1,1}$, N.Q. Li ${ }^{1,2}$, L.H. Liu", ${ }^{1,2}$, Q. Lin ${ }^{1,2}$, Y.G. Li ${ }^{1}$, Y.A. Peng ${ }^{1}$, \\ Z.B. Huang', S.Q. Wu ${ }^{1}$ \\ ${ }^{1}$ Pearl River Fisheries Research Institute, Chinese Academy of Fishery Sciences, \\ Key Laboratory of Fishery Drug Development, Ministry of Agriculture, \\ Key Laboratory of Aquatic Animal Immune Technology, Guangdong Province, Guangzhou 510380, China \\ ${ }^{2}$ Freshwater Aquaculture Collaborative Innovation Center of Hubei Province, Wuhan 430070, China
}

\begin{abstract}
Grass carp reovirus (GCRV) is the causative agent of hemorrhagic disease in infected grass carp. During an outbreak, a mortality rate of up to $85 \%$ can be experienced, thus leading to substantial economic losses.The current understanding of disease pathogenesis is limited, with the distribution and dynamics of replication amongst different GCRV strains in vivo largely unknown. We determined distribution of different GCRV strains in infected grass carp, especially in some neglected tissues, such as the gill, brain, blood and so on. The results showed elevated viral RNA copy numbers in the blood, with some tissues such as the kidney, heart, brain, and bladder exhibiting even higher viral loads following infection with the virulent GCRV-CL strain. Even more interesting is that the brain exhibited the highest viral load, with a copy number of 800,000 following GCRV-CL infection. Overall, this study provides further insight into GCRV viral load distributions following infection and potentially identified some new viral tropism sites to provide a foundation for further studies aimed at characterizing GCRV viral pathogenesis.
\end{abstract}

Key words: distribution, tissue, GCRV, Grass carp

\section{Introduction}

The grass carp reovirus (GCRV) has been recognized as the most pathogenic amongst aquareoviruses and poses as the most serious infectious disease in grass carp. Infection is characterized by hemorrhaging and causes a significant loss of fingerlings during rearing (Jiang et al. 2009). GCRV also produces mortality in the Mylopharyngodon piceus (black carp),
Pseudorasbora parva (stone moroko), the rare minnow Gobiocypris rarus and subclinically in Hypophthalmichthys molitrix (silver carp) and Hemiculter bleekeri (Chinese minnow) (Mathew et al. 2003).

The GCRV is a non-enveloped icosahedral particle containing 11 double-stranded RNA genome segments surrounded by multiple concentric protein capsids (Shao et al. 2011). While considerable fundamental and applied research has been carried out on 
mammalian or human isolates from the genera Orthoreovirus and Rotavirus, the Aquareovirus genus is not as well characterized, with studies focused predominantly on protein molecular and structural biology (Fan et al. 2009). Currently, no effective antiviral treatment against GCRV infection is available (Ma et al. 2011). In an attempt to control the spread of the disease, several inactive vaccines have been developed over the years, yet epidemic outbreaks have still occurred in many freshwater areas in recent years (Chen et al. 2013). Therefore, preventing this infectious disease still remains a great challenge (Cheng et al. 2008) and requires a better understanding of the pathogenic mechanisms underpinning the development of GCRV driven hemorrhagic disease. Currently, the distribution and dynamics of GCRV replication in vivo are largely unknown. In our previous study, we had investigated the viral distribution in some common organs, which often detected in the GCRV study( Liang et al. 2014). The objective of this study was to investigate the viral distribution in some neglected tissues, which in fact had high viral RNA copy numbers.

\section{Materials and Methods}

The GCRV-HZ08 and GCRV-CL strains were obtained from the Pearl River Fishery Research Institute (Guangzhou, China). The GCRV-HZ08 stain was isolated form China's Zhejiang province and GCRV-CL stain was isolated form China's Hunan province, which have different virulence(Wang et al. 2012). Grass carp with a body length of $10 \pm 1.0 \mathrm{~cm}$ and an average weight of $20.0 \pm 1.1 \mathrm{~g}$ were obtained from a GCRV-free zone in NanHai (Guangdong, China).

The grass carp were divided into three groups (60 grass carp per group): (i) grass carp intraperitoneally inoculated with $100 \mu \mathrm{l} \mathrm{GCRV-HZ08;} \mathrm{(ii)} \mathrm{grass} \mathrm{carp}$ intraperitoneally inoculated with $100 \mu \mathrm{l} \mathrm{GCRV-CL;}$ and (iii) grass carp intraperitoneally inoculated with sterile PBS as a negative control group. The grass carps were monitored over three weeks for clinical signs of hemorrhagic disease and sampled.

The brain from the above three groups were sampled at day 7. Duplicate impression smears of $1 \mathrm{~cm}$ in diameter were prepared from each sample. The impression smears were air-dried, fixed with acetone overnight, with the acetone drained off following the overnight incubation and the slides air dried at room temperature. Slides were then washed 3 times with PBS and incubated with rabbit anti-grass carp reovirus antibody $(1: 1000)$ at $37^{\circ} \mathrm{C}$ for $1 \mathrm{~h}$, followed by an incubation with FITC-conjugated goat anti- rabbit IgG (Boster, China) $(1: 100)$ at $37^{\circ} \mathrm{C}$ for $1 \mathrm{~h}$. The smears were then examined using a fluorescence microscope.

Three grass carp from each above group were sampled from the 1st day to the 14th day, then on days 21 and 28 post treatment, with all samples stored at $-80^{\circ} \mathrm{C}$ until use.Viral RNA copy number were measured by quantitative real-time RT-PCR (qRT-PCR) as previously described (Liu Baoqin, 2013). Briefly, total RNA was extracted using Trizol reagent (Invitrogen, NY, USA) according to the manufacturer's protocol and the isolated RNA reverse transcribed using a First Strand cDNA Synthesis Kit (Takara, Japan). The generated cDNA was examined using Premix Ex TaqTM (Perfect Real Time) (Takara, Japan) to assay samples with the following GCRV S7 gene-specific primers: 5'-ccaggaatcaatagcaatc-3' (forward) and 5'-cctgatataatcgctcttc-3' (reverse) and the internal probe: 5'-cgataaccaccactacggctg-3'. The probe was labeled on the 5' end with FAM and on the 3' end with Eclipse. To estimate viral replication, viral-specific mRNA expression was measured by qRT-PCR and expressed as the number of RNA copies per mg of tissue. The results were analyzed using Applied Biosystems 7500 software.

Experimental data were expressed as a mean \pm SD. The data was analyzed via one-way ANOVA analysis and Student's t-test using SPSS 13.0 statistical software.

\section{Results}

The brain tissue impression smears were fixed and incubated with rabbit anti-grass carp reovirus antibody (1:1000), followed by FITC-conjugated goat anti- rabbit IgG (Boster, China) (1:100). The smears were evaluated using a fluorescence microscope (Fig. 1). The tissue impression smears which grass carp infected with GCRV- CL and GCRV-HZ08, were observed under afluorescence microscopy (Fig. 1A and 1B).

Gill samples were evaluated via qRT-PCR (Fig. 2) and showed a higher viral RNA copy number from the 6th day to the 9th day in the GCRV-CL samples, with the highest number noted on day 7 , with more than 5000 copies noted. The GCEV-HZ08 and PBS control groups had undetectable viral loads.

Viral RNA copy numbers in the heart were evaluated via qRT-PCR (Fig. 3) and showed that grass carp infected with GCRV-CL had higher viral numbers from days 5 to 9 , with the highest number noted on day 7. GCRV-HZ08 infected samples had high viral RNA numbers from day 5 to 7 , with overall viral numbers lower than those found in GCRV-CL samples. 
A

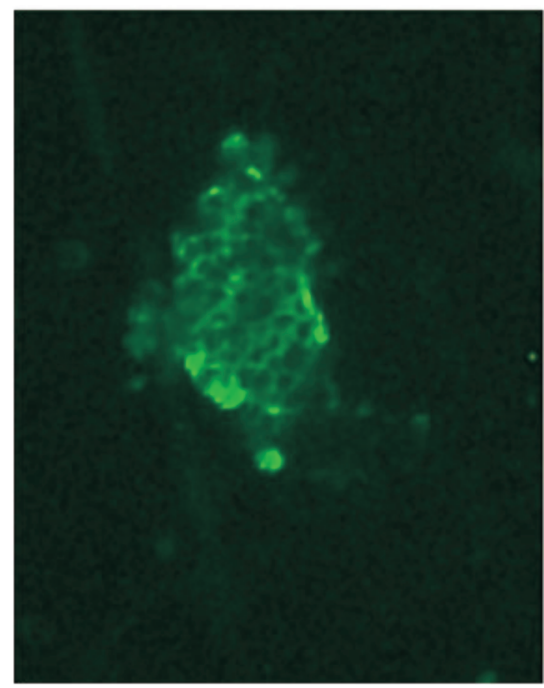

$\mathrm{B}$

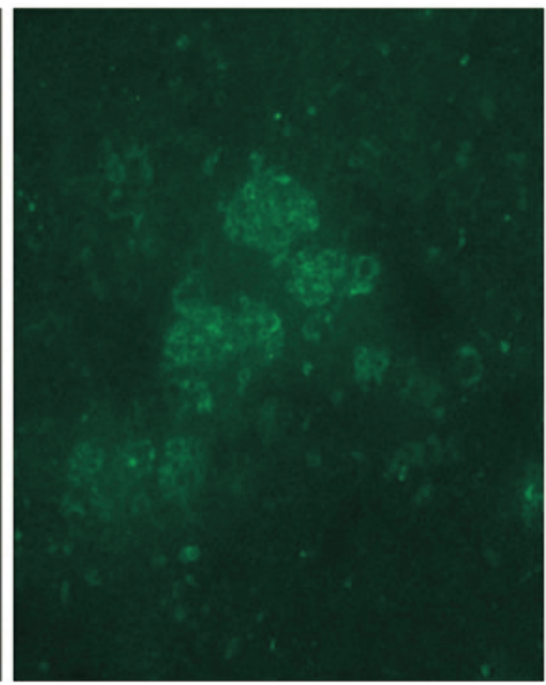

$\mathrm{C}$

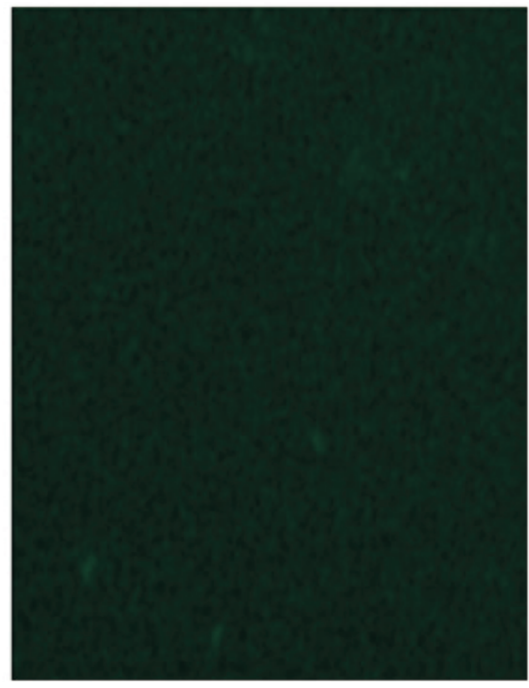

Fig. 1. FAT of brain tissue impression smears on the 7th day after infection. The tissue impression smears were fixed and incubated with rabbit anti-grass carp reovirus antibody (1:1000), followed by FITC-conjugated goat anti- rabbit IgG (Boster, China) (1:100). A: grass carp infected with GCRV-CL; B: grass carp infected with GCRV-HZ08; C: grass carp inoculated with PBS.

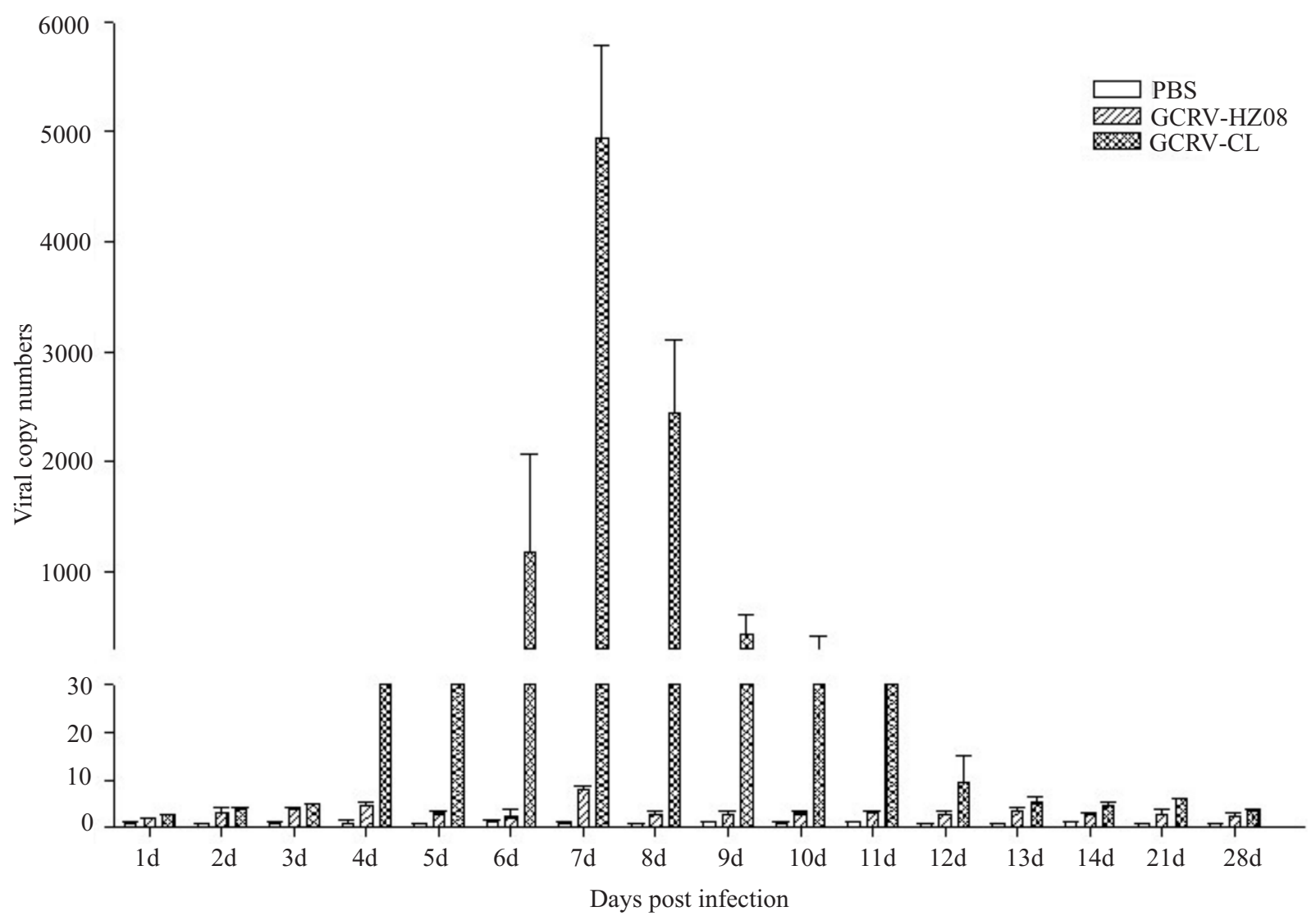

Fig. 2. The viral copy numbers in the gill detected by qRT-PCR. The GCRV-HZ08 and PBS control groups had undetectable viral loads. The GCRV- CL could detect the viral loads. 


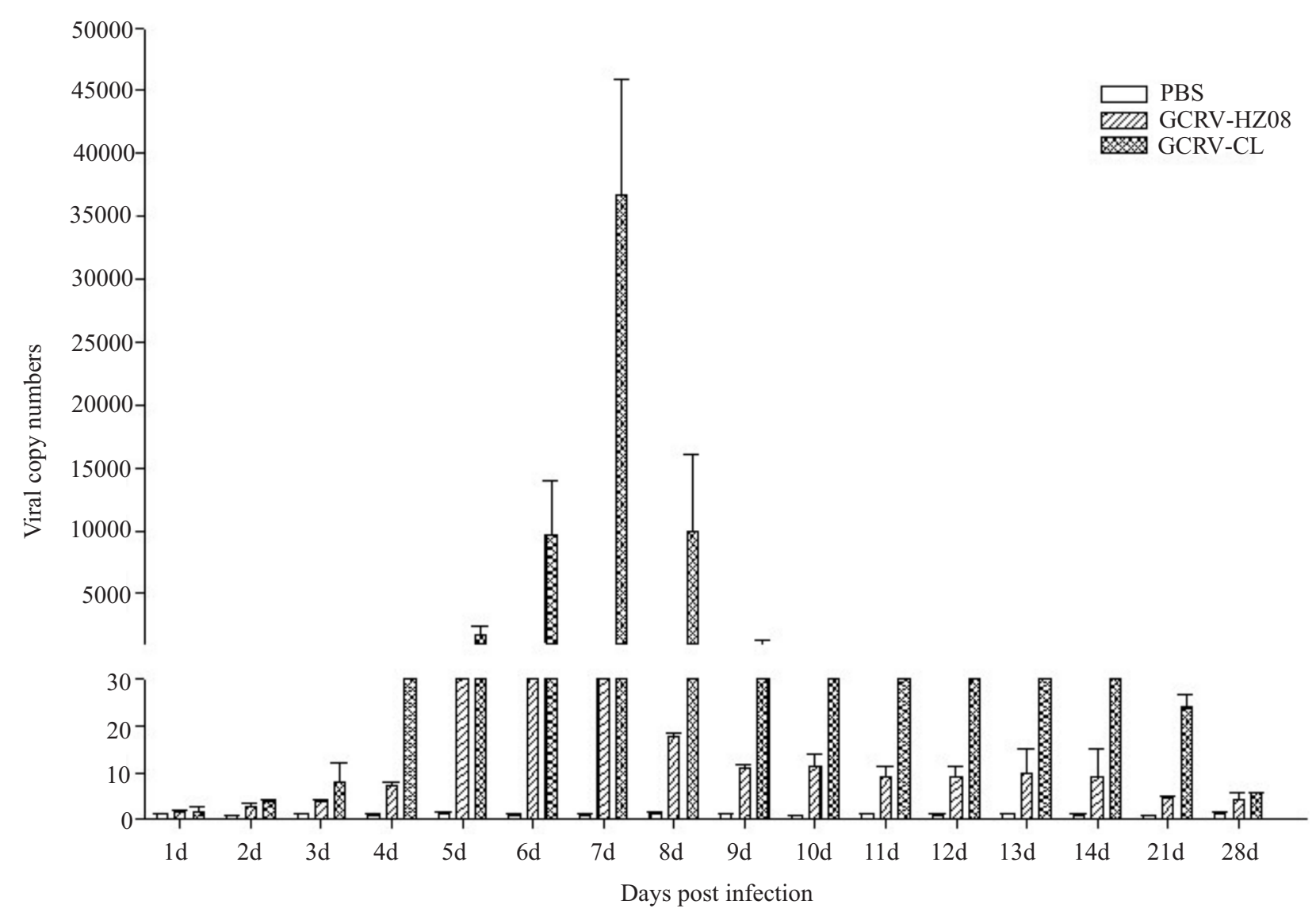

Fig. 3. The viral copy numbers in the heart detected by qRT-PCR.

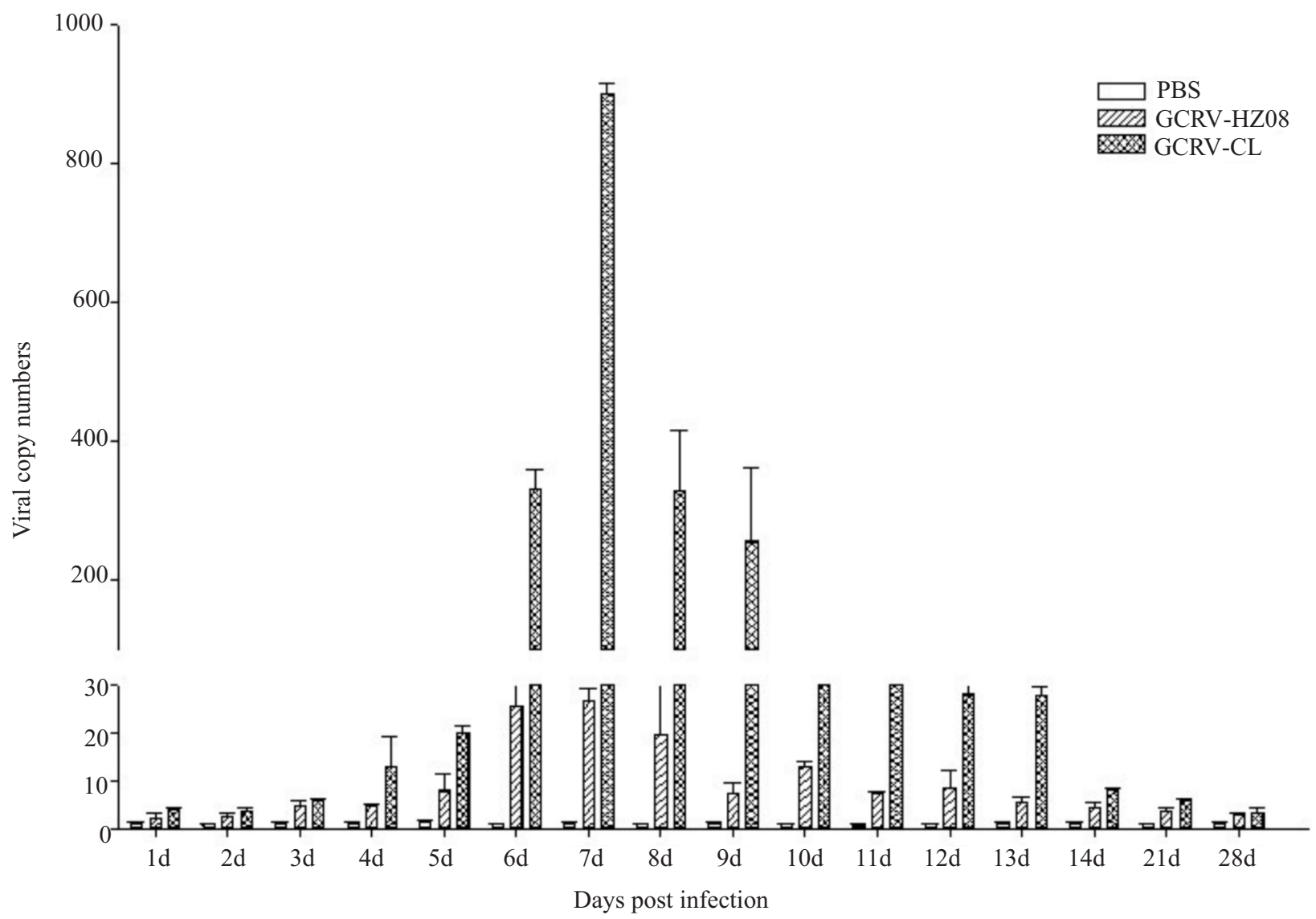

Fig. 4. The viral copy numbers in the blood detected by qRT-PCR. The GCRV-CL samples had higher viral RNA copy numbers than the GCRV-HZ08 samples.The blood had lower viral RNA copy numbers than the other tissues. 


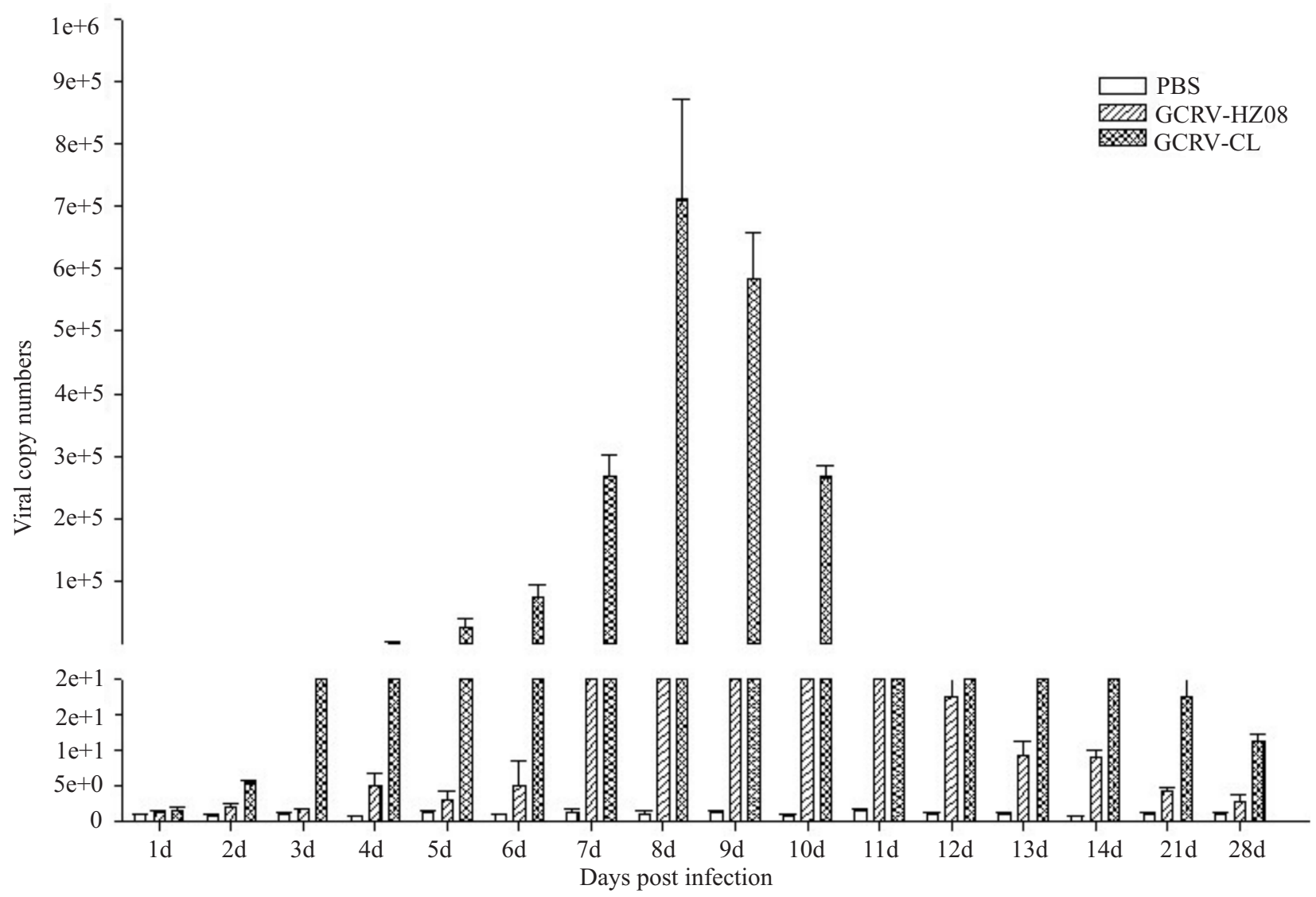

Fig. 5. The viral copy numbers in the brain detected by qRT-PCR. The brain had the highest viral RNA copy numbers considering all the tissues.

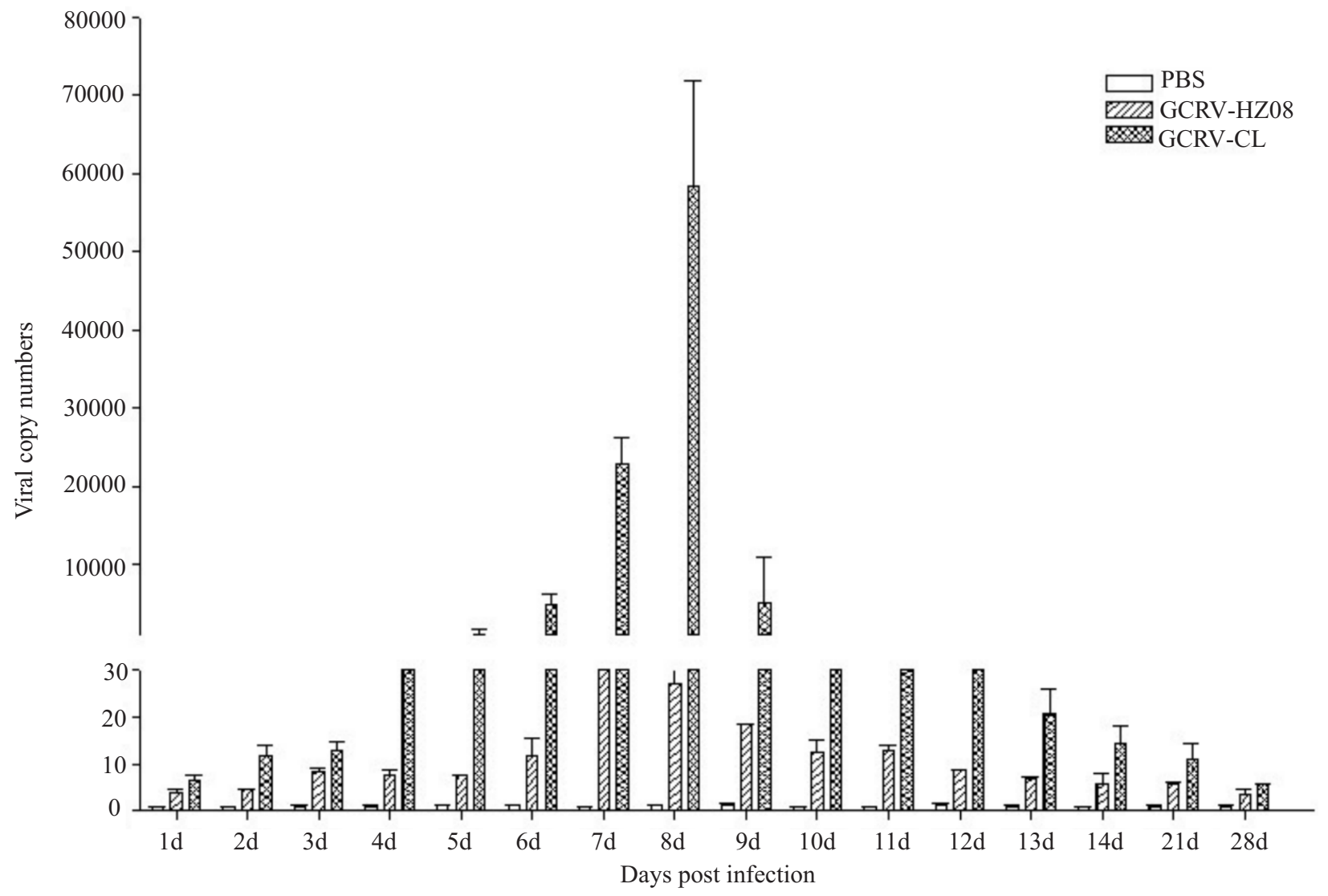

Fig. 6 . The viral copy numbers in the bladder detected by qRT-PCR. The bladder also had high viral RNA copy numbers. 


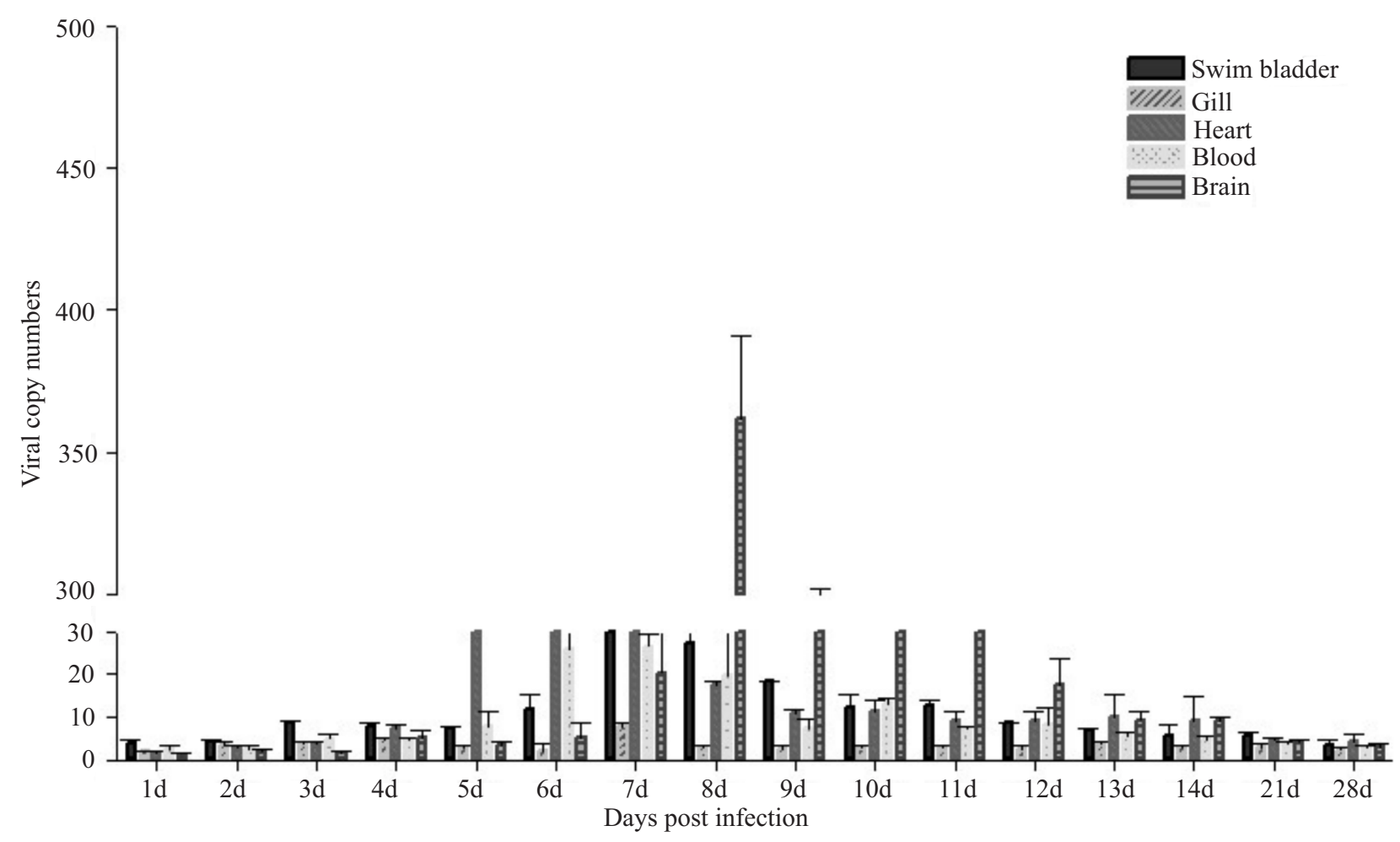

Fig. 7. qRT-PCR viral copy numbers in the GCRV-HZ08 infected samples. All the tissues had lower viral RNA copy numbers than the GCRV-CL infcted samples.

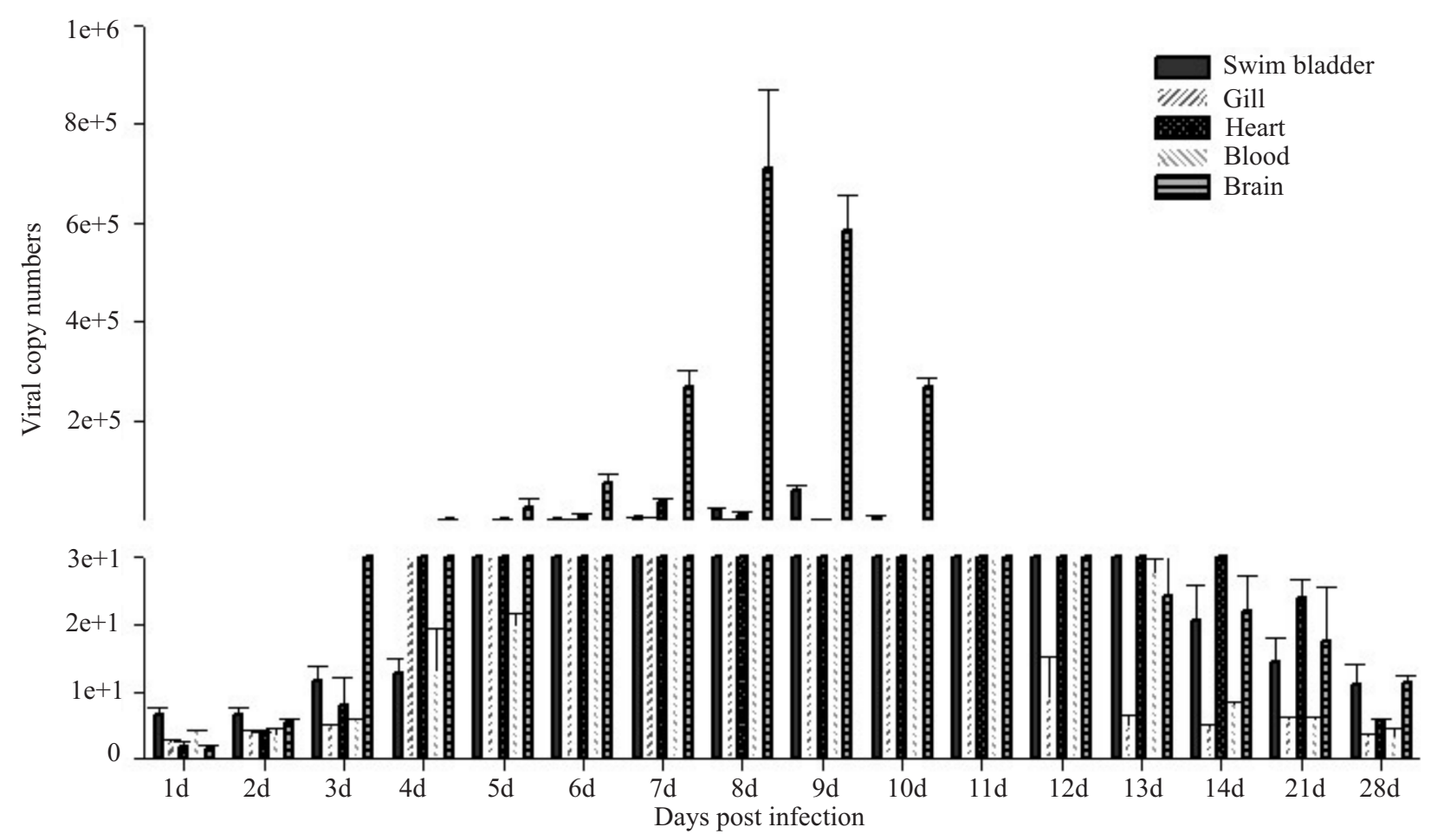

Fig. 8. qRT-PCR viral copy numbers in GCRV-CL infected samples. The GCRV-CL had higher viral RNA copy numbers and relatively long duration. The heart and brain showed the earliest viral presence and displayed the highest viral loads, followed by blood. 
Blood samples were evaluated via qRT-PCR (Fig. 4) and showed elevated viral RNA copy numbers in the GCRV-CL samples from days 6 to 10, with GCRV-HZ08 samples highest on days 6 and 7. And the GCRV-CL samples were higher than those found in the GCRV-HZ08 samples. Relatively, the blood had lower viral RNA copy numbers than the other tissues.

To determine brain viral load, qRT-PCR was utilized (Fig. 5) to show that GCRV-CL samples had higher viral numbers from days 3 to 13 , with copy numbers as high as 800,000 copies noted. GCRV-HZ08 had a higher copy number from day 7 to 10 . When incestigated the grass carp, the brain was always neglected. But in our study, the brain had the highest viral RNA copy numbers considering all the tissues.

Bladder viral RNA copy numbers were evaluated via qRT-PCR (Fig. 6). The GCRV-CL infected grass carp had higher viral numbers from days 5 to 9 , with GCRV-HZ08 infected samples only exhibiting a higher viral load on day 7. The bladder also had relatively high viral RNA copy numbers, especially the GCRV-CL strains.

The results showed that the viral RNA copy numbers in GCRV-HZ08 infected samples were highest overall in the brain on day 8 , with the brain having the longest period of time with an elevated viral load (Fig. 7). The heart was the earliest location to exhibit a high viral load, followed by the blood and bladder. And all the tissues had lower viral RNA copy numbers than those of the GCRV-CL strain.

GCRV-CL infected sample exhibited the highest viral RNA copy numbers in the brain at day 8 and lasted for a period of 6 days (Fig. 8). Overall, the heart and brain showed the earliest viral presence and displayed the highest viral loads, followed by blood. The GCRV-CL had higher viral RNA copy numbers and relatively had long duration.

\section{Discussion}

Based on the inner capsid protein (VP6), Rotaviruses are classified into seven distinct groups: A to G. Groups A, B, and C are associated with acute gastroenteritis in humans and animals, while groups $\mathrm{D}, \mathrm{E}, \mathrm{F}$, and $\mathrm{G}$ have been detected only in animals (Collins et al. 2010). GCRV was first isolated in 1983 and is characterized by severe hemorrhagic disease, with approximately $85 \%$ mortality in fingerling and yearling grass carp (Cyenopharyngodon idellus) in China. The GCRV is classified to species Aquareovirus-C in the genus Aquareovirus, which was thought to be most pathogenic aquareovirus among all the isolates reported to date (Wen et al. 2013). Despite extensive investigation in the past 20 years, the pathogenic mechanisms by which attenuated strains and virulent strain can lead to hemorrhagic death in grass carp have not been well characterized, with a lack of information pertaining to GCRV tissue distributions a potentially important piece.

Reoviruses are divided into virulent strains and attenuated strains. Under experimental conditions, the virulent strain alone was able to induce disease, while the attenuated virus was not (Martella et al. 2007). In this study, we used two GCRV stains: GCRV-CL and GCRV-HZ08, in our previous study showing GCRV-CL to induced hemorrhagic disease, while the GCRV-HZ08 strain did not (Liang et al. 2014). That maybe due to the GCRV-HZ08 strain has been attenuated following cellular passaging, while the GCRV-CL strain remaining more virulent.

In the present study, some neglected tissues or organs from the infected grass carp were sampled from the 1st day to the 28th day, including the gill, bladder, heart, brain and blood. The viral RNA copy numbers were evaluated via GRT-PCR, with a threshold cycle $(\mathrm{Ct})$ lower than $35(\mathrm{Ct}<35$; copy $>20)$ were treated as positive. In nature, GCRV infected grass carp always show swimming abnormalities, violently circle or outliers alone. The GCRV-CL strain infected grass carp showed very high copy rates compared with the HZ08 strain. For example, the GCRV-HZ08 had undetectable viral loads while the GCRV- CL with more than 5000 copies in the blood. And all the tissues infected by GCRV- CL had higher viral RNA copy numbers than the GCRV-HZ08 strain. The GCRV-CL not only had higher viral RNA copy numbers and but also had long duration. Our findings showed the highest viral load to occur in the brain, with the highest copy number of 800,000 noted in GCRV-CL infected samples. This may be attributed to GCRV massive enrichment or even replication in the brain, possibly explaining the ataxia and violent circling noted in infected carp.

Internally, hemorrhaging may occur throughout the musculature, liver, spleen, kidney, and intestines (Jing et al. 2013), thus sampling sites usually consist of the intestine, muscle, spleen, kidney or liver. However, in the present study, we sampled tissues such as the gill, heart, blood, brain, and bladder. To our surprise, all the different tissues had high viral RNA copy numbers except for the blood. In our previous study, the highest viral load was seen in the kidney, with a copy numbers of 25,000 . In this study, the brain greatly exceeded the viral load seen in the kidney, with the heart and bladder possibly serving as viral tropism organs due to their elevated viral loads. The heart was the earliest tissue to exhibit an elevated viral 
load following infection, which may be attributed to the heart being central in the circulatory system and serving as a primary reservoir for circulating GCRV.

\section{Conclusion}

In conclusion, the results obtained in this study provide further insight into GCRV viral load distributions following infection. Our findings also implicated some new viral tropism sites, thus providing a foundation for further studies aimed at understanding GCRV viral pathogenesis.

\section{Acknowledgments}

This project was supported by the PhD Start-up Fund of Natural Science Foundation of Guangdong Province (Grant No. 2015A030310269), the Special Scientific Research Funds for Central Non-profit Institutes, Chinese Academy of Fishery Sciences (Grant No. 2015B05XK02), and the Medjaden Academy $\&$ Research Foundation for Young Scientists (Grant No. MJR20140021) and the earmarked fund for the China Agriculture Research System (Grant No. CARS-46).

\section{References}

Chen C, Sun X, Liao L, Luo S, Li Z, Zhang X, Wang Y, Guo Q, Fang Q, Dai, H (2013) Antigenic analysis of grass carp reovirus using single-chain variable fragment antibody against IgM from Ctenopharyngodon idella. Sci China Life Sci 56(1): 59-65.

Cheng L, Fang Q, Shah S, Atanasov IC, Zhou ZH (2008) Subnanometer-resolution structures of the grass carp reovirus core and virion. J Mol Biol 382(1): 213-222.

Collins PJ, Martella V, Sleator RD, Fanning S, Shea H (2010) Detection and characterisation of group
A rotavirus in asymptomatic piglets in southern Ireland. Arch Virol 155(8): 1247-1259.

Fan C, Zhang L, Lei CF, Fang Q (2009) Expression and identification of inclusion forming-related domain of NS80 nonstructural protein of grass carp reovirus. Virol Sini 24(3): 194-201.

Jiang YL (2009) Hemorrhagic Disease of Grass Carp Status of Outbreaks, Diagnosis, Surveillance, and Research. Isr J Aquaclt-Bamid 61(3): 188-197.

Jing HL, Zhang LF, Fang ZZ, Xu LP, Zhang M,Wang N, Jiang YL, Lin XM (2013) Detection of grass carp reovirus (GCRV) with monoclonal antibodies. Arch Virol 159(4): 649-55.

Liang HR, L YG, Zeng WW, Wang YY, Wang Q, Wu SQ (2014) Pathogenicity and tissue distribution of grass carp reovirus after intraperitoneal administration. Virol J 11(1): 173-178.

Liu BQ, Zeng WW, Wang Q, Zhang LS, Wang YY, Shi CB, Wu SQ (2013) Development of a fluorescent quantitative polymerase chain reaction technique for detection of grass carp reovirus HZ08 strain. J Fishery Sci of China 19: 329-335.

Ma J, Wang WM, Zeng LB., Fan YD, Xu J, Zhou Y (2011) Inhibition of the replication of grass carp reovirus in CIK cells with plasmid-transcribed shRNAs. J Virol Methods 175(2): 182-187.

Martella V, Banyai K, Lorusso E, Decaro N, Bellacicco A, Desario C, Corrente M, Greco G, Moschidou P, Tempesta M., Arista, S., Ciarlet, M., Lavazza, A, Buonavoglia C (2007) Genetic heterogeneity in the VP7 of group $\mathrm{C}$ rotaviruses. Virology 367(2): 358-366.

Mathew E, LukeAndere E (2003) Molecular, physical, and clinical evidence that golden shiner virus and grass carp reovirus are variants of the same virus. J Aquat Anim Health 15(4): 257-263.

Shao L, Sun X, Fang Q (2011) Antibodies against outer-capsid proteins of grass carp reovirus expressed in E. coli are capable of neutralizing viral infectivity. Virol J 8(1): 347-349.

Wen DW, Yan LY, Shao L, Guo H, Li XM, Fang Q (2013) Aquareovirus protein VP6 colocalizes with NS80 protein in infected and transfected cells. Virol J 10(1): 125-133.

Wang Q, Zeng WW, Liu C, Zhang C, Wang YY, Shi CB, Wu SQ (2012) Complete genome sequence of a reovirus isolated from grass carp, indicating different genotypes of GCRV in China. J Virol 86 (22): 12466-12466. 